\title{
Evolution of the single-hole spectral function across a quantum phase transition in the anisotropic-triangular-lattice antiferromagnet
}

\author{
So Takei ${ }^{1}$, Chung-Hou Chung ${ }^{1}$, and Yong Baek Kim ${ }^{1,2}$ \\ ${ }^{1}$ Department of Physics, University of Toronto, Toronto, Ontario M5S 1A7, Canada \\ ${ }^{2}$ School of Physics, Korea Institute for Advanced Study, Seoul 130-722, Korea
}

(Dated: November 12, 2018)

\begin{abstract}
We study the evolution of the single-hole spectral function when the ground state of the anisotropic-triangular-lattice antiferromagnet changes from the incommensurate magneticallyordered phase to the spin-liquid state. In order to describe both of the ground states on equal footing, we use the large- $N$ approach where the transition between these two phases can be obtained by controlling the quantum fluctuations via an 'effective' spin magnitude. Adding a hole into these ground states is described by a $t-J$ type model in the slave-fermion representation. Implications of our results to possible future ARPES experiments on insulating frustrated magnets, especially $\mathrm{Cs}_{2} \mathrm{CuCl}_{4}$, are discussed.

PACS numbers: 75.10.Jm, 71.10.Hf, 79.60.-i
\end{abstract}

\section{INTRODUCTION}

The physics of geometrically frustrated quantum magnetic systems has been an arena for discovery of new and interesting phenomena both on the theoretical and experimental fronts. Much of the effort has been sparked by Anderson's proposal of the exotic resonating valence bond (RVB) spin-liquid phase $\frac{1.2}{2}$ and the belief that frustrated systems are likely to possess RVB spin-liquid-like ground states in two dimensions. Theoretical efforts in the past have explored a variety of two-dimensional frustrated systems 3.4 .5 . The discovery of superconductivity ${ }^{6}$ in $\mathrm{Na}_{0.35} \mathrm{CoO}_{2} \cdot 1.3 \mathrm{H}_{2} \mathrm{O}$ has also raised the hope that the RVB could be the cause of superconductivity in certain frustrated magnetic systems.

Recent theoretical works on the insulating anisotropictriangular-lattice antiferromagnet 7.8.9.10.11 $^{\text {have been }}$ particularly motivated by the experimental discovery of the fractionalized spin-liquid phase in $\mathrm{Cs}_{2} \mathrm{CuCl}_{4}{ }^{12.13}$, where spin- $1 / 2$ moments of $\mathrm{Cu}$ antiferromagnetically interact with each other in the layers of anisotropic triangular lattices. The experiment has shown that, with a sufficiently high magnetic field within its ordering plane, the compound looses its long-ranged cycloid order and enters the spin-liquid phase $\mathrm{12}^{12,13}$. In this phase, the dynamical spin structure factor shows a broad profile characteristic of fractionalization of spin-1 magnons into a pair of spin- $1 / 2$ spinons 12.13 .

A useful description of this transition can be obtained in the large- $N$ approach 8.14 .15 .16 .17 .18 .19 .20 .21 to the antiferromagnetic Heisenberg model on the anisotropic triangular lattice. Here the spin $\mathrm{SU}(2)$ symmetry is generalized to $\operatorname{Sp}(N)$ and the large- $N$ limit of the model is investigated. In this approach, the quantum fluctuations are controlled by the parameter $\kappa$ that is the $\operatorname{Sp}(N)$ analog of the spin magnitude. The resulting phase diagram contains both the incommensurate long-ranged order (LRO) phase ${ }^{22}$ (this corresponds to the cycloid order) and the spin-liquid state ${ }^{23}$ in the large and small $\kappa$ limits, respectively ${ }^{8.18}$. Thus one may expect that $1 / \kappa$ plays a role similar to the in-plane magnetic field in $\mathrm{Cs}_{2} \mathrm{CuCl}_{4}$. Indeed the spin structure factor of the spin-liquid state computed in the large- $N$ theory ${ }^{9.10}$ looks very similar to that observed in the neutron scattering experiments on $\mathrm{Cs}_{2} \mathrm{CuCl}_{4}{ }^{12.13}$.

To this date, experimental evidence of the RVB spinliquid phase in the anisotropic triangular lattice has been obtained only in neutron scattering experiments on $\mathrm{Cs}_{2} \mathrm{CuCl}_{4}{ }_{4}^{12,13}$. Thus it is desirable to have independent experimental confirmations of these results. One such experiment may be the angle-resolved photoemission spectroscopy (ARPES) that measures the electron spectral function. In particular, the result of ARPES on an insulating compound at half-filling corresponds to measuring the single-hole spectral function in a given ground state of the insulator 24 .

In this paper, we investigate the evolution of the singlehole spectral function across the transition between the incommensurate LRO phase and the spin-liquid phase. Previously the single-hole spectral function in the LRO phase was investigated by taking into account the interaction of the hole with the spin waves 25.26 .27 .28 . The well-defined low-energy quasiparticle peak was found in these studies. This is due to the fact that small lowenergy density of states of the magnons leads to only a weak perturbation to the quasiparticle coherence at low energy. While this calculation is enough to describe the spectral function deep inside the ordered phase, an additional ingredient may have to be considered close to the transition toward the nearby spin-liquid state where the spinons are deconfined ${ }^{23}$. The confinement of the spinons in the LRO phase is a low energy phenomenon and it is expected that the confinement energy scale will be very small near the transition. Therefore, it is expected that there will be spinon excitations at intermediate to high energies near the transition and they may affect the coherence of the hole motion. For example, the neutron scattering experiments on the magnetically ordered phase of $\mathrm{Cs}_{2} \mathrm{CuCl}_{4}$ found the spinon continuum as well as the magnon peak, implying that this ordered phase is very 
close to the transition ${ }^{12.13}$. Indeed the dominant interaction near the transition may be the spinon-hole interaction since the magnons are relatively suppressed close to the transition.

We use the results of the large- $N$ theory to describe the transition between two ground states at half-filling. In this formulation, as will be explained later, the spinons appear at the mean field level even in the LRO phase ${ }^{8.14 .15 .16 .17}$. Even though this is an artifact of the mean field theory and the low energy spinons are confined due to the fluctuation effects beyond the mean field theory ${ }^{14.15 .16 .17}$, we can still use these spinons at intermediate to high energies to mimic the situations near the transition as described above. The motion of the singlehole is incorporated via a $t$ - $J$ type model in the slave fermion representation ${ }^{27}$. Our computation takes into account the multiple interactions between the motion of the hole and the spin background, but does not include the vertex corrections. In principle, the latter approximation could lead to an underestimation of the coherence in the motion of the hole. Comparison to the exact diagonalization study ${ }^{29}$, however, suggests that the latter approximation does not change the overall structure of the spectral function in a significant fashion.

Our results indicate that the single-hole spectral function still shows a well-defined low-energy quasiparticle peak in the incommensurate LRO phase with a visible dispersion in momentum space. This is due to the fact that the density of states of spinons becomes smaller as the energy scale is decreased and, as a result, does not strongly disturb the low energy motion of the hole. As the transition to the spin-liquid state is approached, the spectral weight of the quasiparticle peak is decreased and the incoherent continuum at intermediate to high energies grows. Eventually the quasiparticle peak disappears at the transition. In the spin-liquid state, the single-hole spectral function only shows a broad incoherent continuum with little dispersion, which can be interpreted as a consequence of spin-charge separation.

The rest of the paper is organized as follows. In section II, we describe the formalism used to compute the single-hole spectral function and explain the approximation schemes used in this work. In section III, the numerical results on the single-hole spectral function in various phases are discussed. Finally, we summarize our results and conclude in section IV.

\section{FORMULATION OF THE PROBLEM}

At half-filling, the quantum ground states of the Mott insulator on the anisotropic triangular lattice are described by the Heisenberg model:

$$
H_{J}=J_{1} \sum_{\langle i, j\rangle} \mathbf{S}_{i} \cdot \mathbf{S}_{j}+J_{2} \sum_{\langle\langle i, j\rangle\rangle} \mathbf{S}_{i} \cdot \mathbf{S}_{j},
$$

where the first and second terms represent the nearest and the next-nearest neighbour Heisenberg couplings, re-

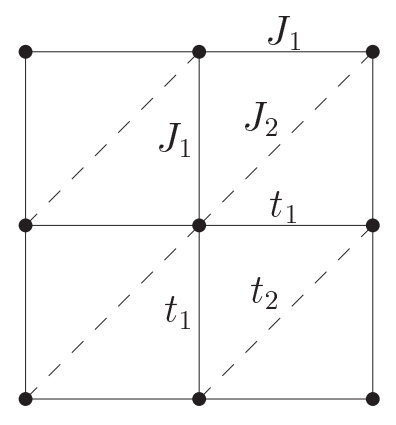

FIG. 1: Anisotropic triangular lattice with two types of bond. The lattice is modelled by a square lattice with an additional interaction along one of the diagonals. The nearest neighbour coupling is associated with subscript 1 and the next-nearest neighbour coupling is associated with subscript 2 .

spectively (see Fig.1). The motion of the single hole in a given ground state of the Mott insulator can be studied by adding the kinetic energy terms of the electrons;

$$
H_{t}=-t_{1} \sum_{\langle i, j\rangle}\left(c_{i \sigma}^{\dagger} c_{j \sigma}+\text { h.c. }\right)-t_{2} \sum_{\langle\langle i, j\rangle\rangle}\left(c_{i \sigma}^{\dagger} c_{j \sigma}+\text { h.c. }\right),
$$

where $c_{i \sigma}^{\dagger}$ is the creation operator of the electrons with spin $\sigma=\uparrow, \downarrow$ (repeated indices are summed) and it is assumed that there is no doubly occupied site in the Hilbert space to take into account the strong correlation inherent in the Mott insulator.

\section{A. Ground States of the Mott Insulator via the Large- $N \operatorname{Sp}(N)$ Heisenberg Model}

A useful formulation of the Heisenberg model in Eq 1 can be obtained from a bosonic representation of the $\mathrm{SU}(2)$ spin via $S_{i}^{a}=\frac{1}{2} b_{i \sigma}^{\dagger} \tau_{\sigma \sigma^{\prime}}^{a} b_{i \sigma^{\prime}}$, where $\tau^{a}(a=x, y, z)$ are the Pauli matrices and $b_{i \sigma}$ is the canonical bosonic operator. In addition, the constraint $b_{i \sigma}^{\dagger} b_{i \sigma}=n_{b}=2 S$ must be imposed at each site, where $S$ is the magnitude of the spin. In order to study this model on frustrated lattices in a controlled fashion and to obtain results that are non-perturbative in the coupling constants, we consider the $\operatorname{Sp}(N)$ generalization ${ }^{14.15 .16 .17}$ of the physical spin $\mathrm{SU}(2) \simeq \mathrm{Sp}(1)$. This can be achieved by introducing $N$ flavours of bosons at each site, $b_{i \alpha}$, where $\alpha=(n, \sigma)$ and $n=1, \ldots, N$ is the flavour index. Here again the boson number at each site, $n_{b}=b_{i \alpha}^{\dagger} b_{i \alpha}$, must be fixed. The resulting $\operatorname{Sp}(N)$ Heisenberg model is given by ${ }^{14,15,16.17}$

$$
H_{\mathrm{Sp}(N)}=-\sum_{i>j} \frac{J_{i j}}{2 N}\left(\mathcal{J}_{\alpha \beta} b_{i \alpha}^{\dagger} b_{j \beta}^{\dagger}\right)\left(\mathcal{J}_{\gamma \delta} b_{i \gamma} b_{j \delta}\right),
$$

where $J_{i j}=J_{1}$ and $J_{2}$ on the nearest and next-nearest neighbour links, respectively. Here $\mathcal{J}_{\alpha \beta}=-\mathcal{J}_{\beta \alpha}$ is a generalization of the $\mathrm{SU}(2)$ antisymmetric tensor $\epsilon_{\sigma \sigma^{\prime}}=$ 


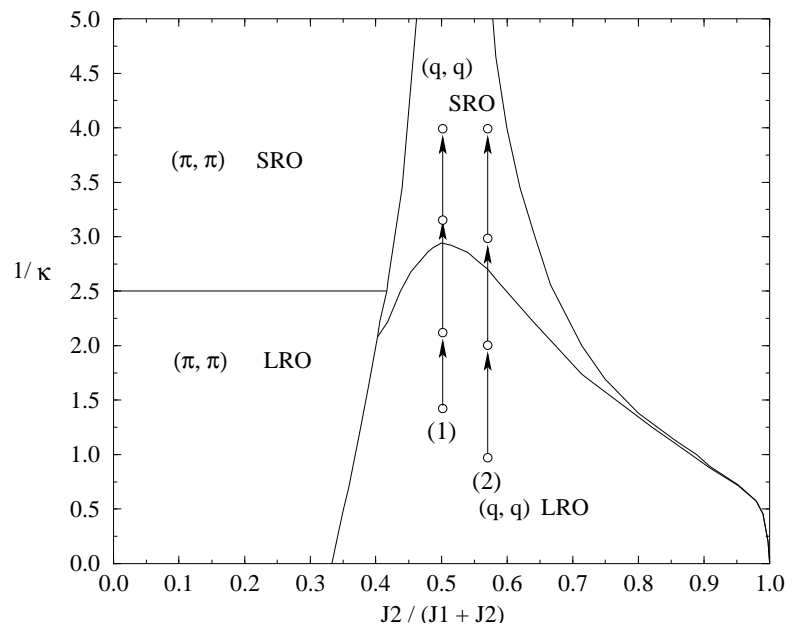

FIG. 2: The zero temperature phase diagram $^{8}$ of the $\operatorname{Sp}(N)$ Heisenberg antiferromagnet on the anisotropic triangular lattice at large $N .1 / \kappa$ represents the strength of quantum fluctuations. Single hole spectral functions are evaluated at each of the ground states denoted by the circles along paths (1) and (2). See text for details.

$-\epsilon_{\sigma^{\prime} \sigma}$ and is given by a $2 N \times 2 N$ matrix that has $N$ copies of $\epsilon$ along its center block diagonal. The matrix is zero elsewhere.

We consider the large- $N$ limit of this model at a fixed boson density per flavour, $\kappa=n_{b} / N$. Notice that $\kappa=2 S$ when $N=1$. Thus $\kappa$ is the $\operatorname{Sp}(N)$ analog of $2 S$ and controls the strength of quantum fluctuations. The large$N$ mean-field theory of this model has been analyzed 8 and the phase diagram at large- $N$ has been obtained as a function of $\kappa$ and $J_{2} /\left(J_{1}+J_{2}\right)$ (see Fig. 2$)^{8}$. For large values of $\kappa$, quantum fluctuations are small so that the LRO states arise by breaking the global $\operatorname{Sp}(N)$ symmetry. In the small $\kappa$ limit, strong quantum fluctuations lead to paramagnetic states with only short-ranged order (SRO). In the phase diagram of Fig.2, the ordering wavevector of the LRO states (and the corresponding SRO phases) are labeled by the ordering wavevector $\mathbf{q}=\left(q_{1}, q_{2}\right)$ in two dimensions. It has been known that $(\pi, \pi)$ SRO phase develops the valence-bond-solid order upon inclusion of singular fluctuations beyond the mean-field theory and the Berry phase effect ${ }^{4.8}$. In this paper, we will consider the transition between the incommensurate $(q, q)$ LRO and the $(q, q)$ SRO phases that respectively correspond to the cycloid-ordered and the translationally-invariant spin-liquid phases discovered in the experiment involving $\mathrm{Cs}_{2} \mathrm{CuCl}_{4}{ }^{12.13}$.

Now, for future reference, some explanation of the large- $N$ mean-field theory is in order. The presence of the LRO can be described by the condensation of bosons, $\left\langle b_{i}^{n \sigma}\right\rangle=\sqrt{N} \delta_{1}^{n} x_{i}^{\sigma}$, where the $c$-number field $x_{i}^{\sigma}$ quantifies the condensate. Thus, in the LRO phase, it is convenient to parametrize the bosonic fields as

$$
b_{i}^{n \sigma}=\left(\begin{array}{c}
\sqrt{N} x_{i}^{\sigma} \\
b_{i}^{m \sigma}
\end{array}\right)
$$

where $m=2, \ldots, N$. On the other hand, none of the bosons is condensed in the SRO phase. After decoupling the quartic boson interaction in Eq[3 by the HubbardStratonovic fields $Q_{i j}$, the mean-field Hamiltonian can be obtained as 14,15,16,17.18

$$
\begin{aligned}
H_{M F} & =\sum_{i>j}\left[\frac{N}{2} J_{i j} Q_{i j}\right. \\
& \left.-\frac{\epsilon_{\sigma \sigma^{\prime}}}{2} J_{i j} Q_{i j}\left(N x_{i}^{\sigma} x_{j}^{\sigma^{\prime}}+\sum_{m=2}^{N} b_{i}^{m \sigma} b_{j}^{m \sigma^{\prime}}\right)+\text { h.c. }\right] \\
& +\sum_{i} \lambda_{i}\left(N\left|x_{i}^{\sigma}\right|^{2}+\sum_{m=2}^{N} b_{i, m \sigma}^{\dagger} b_{i}^{m \sigma}-n_{b}\right)
\end{aligned}
$$

where $Q_{i j}=\frac{1}{N}\left\langle\mathcal{J}_{\alpha \beta} b_{i \alpha}^{\dagger} b_{j \beta}^{\dagger}\right\rangle$ at the saddle of the corresponding action. Here the last term of Eq 5 takes care of the constraint on the boson number at each site and $\lambda_{i}$ is the Lagrange multiplier.

At the mean-field level, $Q_{i j}=Q_{1}$ and $Q_{2}$ on the nearest and the next-nearest neighbour links, and $\lambda$ is a siteindependent constant. In the LRO phase, the boson condensate fraction has the form

$$
x_{i \uparrow}=x e^{i \mathbf{Q} \cdot \mathbf{r}_{i}}, \quad x_{i \downarrow}=-i x^{*} e^{-i \mathbf{Q} \cdot \mathbf{r}_{i}},
$$

where $\mathbf{q}=2 \mathbf{Q}$ is the magnetic ordering wavevector. In the mean-field theory, $Q_{1}, Q_{2}, x, \lambda$ and $\mathbf{Q}$ have to be determined self-consistently for a given $\kappa=n_{b} / N$.

The part of the Hamiltonian for the uncondensed bosons or spinons, $b_{i m \sigma}(m=2, \ldots, N)$, can be diagonalized using the Bogoliubov transformation:

$$
H_{M F}^{\prime}=\sum_{\mathbf{k}} \sum_{m=2}^{N} \epsilon_{\mathbf{k}}\left(\alpha_{\mathbf{k} m}^{\dagger} \alpha_{\mathbf{k} m}+\beta_{\mathbf{k} m}^{\dagger} \beta_{\mathbf{k} m}\right),
$$

where

$$
\begin{aligned}
\epsilon_{\mathbf{k}} & =\sqrt{\lambda^{2}-\left|\gamma_{\mathbf{k}}\right|^{2}} \\
\gamma_{\mathbf{k}} & =i\left[J_{1} Q_{1}\left(\sin k_{x}+\sin k_{y}\right)+J_{2} Q_{2} \sin \left(k_{x}+k_{y}\right)\right] \\
& =\left|\gamma_{\mathbf{k}}\right| e^{i \theta_{\mathbf{k}}} .
\end{aligned}
$$

The Bogoliubov quasiparticle operators, $\alpha_{\mathbf{k} m}$ and $\beta_{\mathbf{k} m}$, are given by

$$
\left(\begin{array}{c}
\alpha_{\mathbf{k} m} \\
\beta_{-\mathbf{k} m}^{\dagger}
\end{array}\right)=\left(\begin{array}{cc}
u_{\mathbf{k}} & v_{\mathbf{k}} \\
v_{\mathbf{k}}^{*} & u_{\mathbf{k}}
\end{array}\right)\left(\begin{array}{c}
b_{\mathbf{k} m \uparrow} \\
b_{-\mathbf{k} m \downarrow}^{\dagger}
\end{array}\right)
$$

where

$$
u_{\mathbf{k}}=\frac{1}{\sqrt{2}} \sqrt{\frac{\lambda}{\epsilon_{\mathbf{k}}}+1}, \quad v_{\mathbf{k}}=\frac{e^{-i \theta_{\mathbf{k}}}}{\sqrt{2}} \sqrt{\frac{\lambda}{\epsilon_{\mathbf{k}}}-1} .
$$

Notice that, in the SRO phase, there are no condensed bosons and all the spinons are described by $\mathrm{Eq} 7$ 
It has been known that the presence of unconfined spinons is an artifact of the large- $N$ mean-field theory and these spinons are, in principle, confined at low energies due to fluctuations beyond the mean-field theory 14.15.16.17 Thus the spinons cannot be seen deep inside the LRO phases. The confinement scale below which the confinement occurs, however, can be very small when the transition to the spin-liquid phase is approached. Thus we expect that there exist 'almost'-deconfined (or loosely-confined) spinons at intermediate to high energy scales near the transition. At the transition point, this energy scale should be zero because the spinons are deconfined in the spin-liquid phase. In fact, the experiment on $\mathrm{Cs}_{2} \mathrm{CuCl}_{4}$ reveals the existence of both of the magnon peak and the spinon continuum in the cycloidordered phase, indicating that this system is very close to the transition toward the spin-liquid phase ${ }^{12.13}$. While the confinement phenomenon cannot be captured by the mean-field theory, the existence of the deconfined spinons at the mean-field level can be used to phenomenologically describe the existence of 'almost'-deconfined spinons near the transition. From now on, we will adopt this picture and examine the effect of the 'almost'-deconfined spinons on the motion of the holes.

\section{B. Motion of Single Hole and the Spectral Function}

In order to describe the motion of a single hole in the large- $N$ limit, we use the slave-fermion representation of the electron operator with the flavour index:

$$
c_{i, n \sigma}^{\dagger}=f_{i} b_{i, n \sigma}^{\dagger},
$$

where $f_{i}$ represents the fermionic charge degree of freedom and $b_{i, n \sigma}$ the bosonic spin degree of freedom of the electrons.

The single-hole spectral function can be computed from the single-hole Green's function:

$$
\begin{aligned}
G_{h, \sigma}(\mathbf{r}, \tau) & =\frac{1}{N} \sum_{n} G_{h, n \sigma}(\mathbf{r}, \tau) \\
& =-\frac{1}{N} \sum_{n}\left\langle T_{\tau} c_{n \sigma}^{\dagger}(\mathbf{r}, \tau) c_{n \sigma}(0,0)\right\rangle,
\end{aligned}
$$

where $T_{\tau}$ is the time-ordering operator of the imaginary time. Notice that the physical hole Green's-function is defined as the average of the Green's functions in different flavour channels. Since no flavour degree of freedom is special on physical grounds, this is a legitimate definition of the hole Green's function that turns out to be particularly useful for our purposes.

After analytic continuation, the spectral function can be obtained from

$$
A_{h, \sigma}(\mathbf{k}, \omega)=-\frac{1}{\pi} \operatorname{Im}\left[G_{h, \sigma}(\mathbf{k}, \omega+i \delta)\right],
$$

where $G_{h, \sigma}(\mathbf{k}, \omega)$ is the single-hole Green's function in Fourier space.
At the mean-field level, the single-hole Green's function can be written as

$$
G_{h, \sigma}(\mathbf{r}, \tau) \approx-\frac{1}{N} \sum_{n} G_{f}(\mathbf{r}, \tau) G_{b, n \sigma}(\mathbf{r}, \tau),
$$

where

$$
\begin{aligned}
G_{f}(\mathbf{r}, \tau) & =-\left\langle T_{\tau} f(\mathbf{r}, \tau) f^{\dagger}(0,0)\right\rangle \\
G_{b, n \sigma}(\mathbf{r}, \tau) & =-\left\langle T_{\tau} b_{n \sigma}^{\dagger}(\mathbf{r}, \tau) b_{n \sigma}(0,0)\right\rangle .
\end{aligned}
$$

In Fourier space, we get

$$
\begin{aligned}
& G_{h, \sigma}(\mathbf{k}, \omega) \\
= & -\frac{1}{N} \sum_{n} \sum_{\mathbf{q}} \int d \nu G_{b, n \sigma}(\mathbf{k}-\mathbf{q}, \omega-\nu) G_{f}(\mathbf{q}, \nu) .
\end{aligned}
$$

Thus the single-hole Green's function is given by the convolution of the fermionic and the bosonic Green's functions.

In this work, we will assume that the injection of the single-hole does not affect the underlying ground state at half-filling. In this case, it may be sufficient to use the bare bosonic Green's-function, $G_{b, n \sigma}^{0}$, in the absence of the hole ${ }^{27}$. On the other hand, the fermionic degree of freedom of the hole will be very much affected by the presence of the excitations in the underlying ground state. We will compute the renormalized fermionic Green's function using the self-consistent Dyson equation ${ }^{27.28}$, where the non-crossing diagrams are summed. Possible effect of the vertex corrections in the fermionic Green's function and Eq14 will be discussed later.

\section{Computation of the Fermion Green's Function}

The proper large- $N$ generalization of the kinetic energy terms of the holes in EqL2 is given by

$$
\begin{aligned}
H_{t} & =-\frac{t_{1}}{N} \sum_{\langle i, j\rangle}\left(f_{i} b_{i, n \sigma}^{\dagger} b_{j, n \sigma} f_{j}^{\dagger}+h . c .\right) \\
& -\frac{t_{2}}{N} \sum_{\langle\langle i, j\rangle\rangle}\left(f_{i} b_{i, n \sigma}^{\dagger} b_{j, n \sigma} f_{j}^{\dagger}+\text { h.c. }\right)
\end{aligned}
$$

Fourier transformation of these terms leads to

$$
H_{t}=-\frac{1}{N} \sum_{\mathbf{k k}^{\prime} \mathbf{q}} \Gamma_{\mathbf{k}-\mathbf{k}^{\prime}} f_{\mathbf{k}} b_{\mathbf{k}^{\prime}, n \sigma}^{\dagger} b_{\mathbf{k}^{\prime}-\mathbf{q}, n \sigma} f_{\mathbf{k}-\mathbf{q}}^{\dagger}
$$

where

$$
\Gamma_{\mathbf{k}}=2\left[t_{1}\left(\cos k_{x}+\cos k_{y}\right)+t_{2} \cos \left(k_{x}+k_{y}\right)\right] .
$$

These hopping terms give rise to the interaction between the hole and the spin background.

In the LRO phase, the existence of the condensed bosons, $\left|\left\langle b_{\mathbf{k}, n \sigma}\right\rangle\right|^{2}=N \delta_{1}^{n}|x|^{2} \delta_{\mathbf{k}, \mathbf{Q}}$, leads to

$$
H_{t}=2 \sum_{\mathbf{k}} \Gamma_{\mathbf{k}-\mathbf{Q}}|x|^{2} f_{\mathbf{k}}^{\dagger} f_{\mathbf{k}}
$$




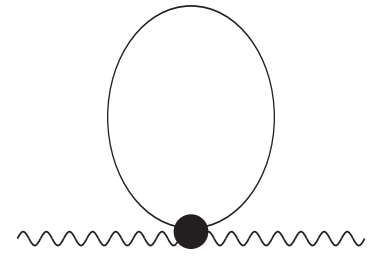

FIG. 3: The self-energy correction of Eq21 The wavy line represents the fermionic propagator and the solid line represents the bosonic propagator.

$$
+\frac{1}{N} \sum_{\mathbf{k} \mathbf{k}^{\prime} \mathbf{q}} \sum_{m=2}^{N} \Gamma_{\mathbf{k}-\mathbf{k}^{\prime}} b_{\mathbf{k}^{\prime}, m \sigma}^{\dagger} b_{\mathbf{k}^{\prime}-\mathbf{q}, m \sigma} f_{\mathbf{k}}^{\dagger} f_{\mathbf{k}-\mathbf{q}}
$$

The second term corresponds to the interaction between the uncondensed spinons and the fermion and gives rise to the fermion self-energy corrections.

Let us first consider the perturbative self-energy corrections in $t_{1} / N$ and $t_{2} / N$. The lowest-order correction is given by (see Fig.3)

$$
\Sigma_{1}(\mathbf{k})=2 \sum_{\mathbf{k}^{\prime}} \Gamma_{\mathbf{k}-\mathbf{k}^{\prime}}\left|v_{\mathbf{k}^{\prime}}\right|^{2}
$$

where $(N-1) / N \approx 1$ is used. The next order correction involves the diagram in Fig. 4 and can be computed as

$$
\Sigma_{2}(\mathbf{k}, \omega)=\frac{1}{N} \sum_{\mathbf{k}^{\prime}, \mathbf{q}} f_{\mathbf{k k}^{\prime} \mathbf{q}} G_{f}\left(\mathbf{k}-\mathbf{q}, \omega-\epsilon_{\mathbf{k}^{\prime}}-\epsilon_{\mathbf{q}-\mathbf{k}^{\prime}}\right),
$$

where

$$
f_{\mathbf{k k}^{\prime} \mathbf{q}}=\left|\Gamma_{\mathbf{k}-\mathbf{k}^{\prime}} u_{\mathbf{k}^{\prime}} v_{\mathbf{k}^{\prime}-\mathbf{q}}+\Gamma_{\mathbf{k}+\mathbf{k}^{\prime}-\mathbf{q}} u_{\mathbf{k}^{\prime}-\mathbf{q}} v_{\mathbf{k}^{\prime}}\right|^{2}
$$

and $(N-1) / N^{2} \approx 1 / N$ is used. Notice that, in the perturbative calculation, the bare fermionic Green'sfunction, $G_{f}^{0}$, should be used for $G_{f}$ in $\mathrm{Eq} 22$

In order to take into account the multiple interactions, however, we need to go beyond the perturbative evaluations of the self-energy contributions. In our work, we will determine the renormalized fermion Green's function, $G_{f}$, by summing up diagrams in the non-crossing approximation. This amounts to using the renormalized Green's function in Eq 22 and solving the following Dyson equation for the fermion Green's function.

$$
G_{f}^{-1}(\mathbf{k}, \omega)=\omega+2|x|^{2} \Gamma_{\mathbf{k}-\mathbf{Q}}-\Sigma_{1}(\mathbf{k})-\Sigma_{2}(\mathbf{k}, \omega) .
$$

In the numerical solution of this equation, we set the coefficient of the self-energy correction in EqL22 to be one for convenience (i.e. $1 / N=1$ ). Notice that $2|x|^{2} \Gamma_{\mathbf{k}-\mathbf{Q}}-$ $\Sigma_{1}(\mathbf{k})$ corresponds to the dynamically generated 'band' dispersion for the fermions at the lowest order. In the case of the SRO phase, the same Dyson equation can be used with $x=0$ in Eq24

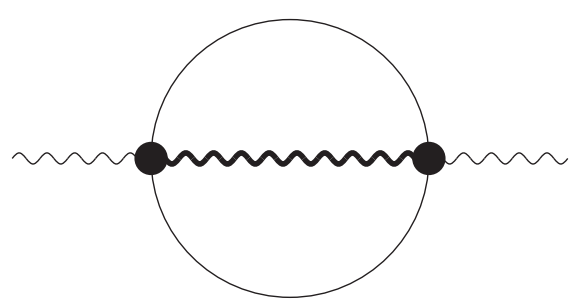

FIG. 4: The self-energy correction of Eq22 The thick wavy line is the exact fermionic propagator. Combined with the contribution from Fig 3 this gives rise to the Dyson equation for the fermionic propagator in Eq[24]

\section{Evaluation of the Single-Hole Spectral Function}

In the LRO phase, the Green's function for the condensed bosons in the Fourier space is given by

$$
\begin{aligned}
& G_{b, 1 \uparrow}(\mathbf{k}, \omega)=-N|x|^{2} \delta_{\mathbf{k},-\mathbf{Q}} \delta(\omega) \\
& G_{b, 1 \downarrow}(\mathbf{k}, \omega)=-N|x|^{2} \delta_{\mathbf{k}, \mathbf{Q}} \delta(\omega) .
\end{aligned}
$$

On the other hand, the Green's function for the uncondensed bosons are obtained as (for both $\sigma=\uparrow, \downarrow$ )

$$
G_{b, m \sigma}(\mathbf{k}, \omega+i \delta)=\frac{\left|v_{\mathbf{k}}\right|^{2}}{\left(\omega-\epsilon_{\mathbf{k}}+i \delta\right)}-\frac{u_{\mathbf{k}}^{2}}{\left(\omega+\epsilon_{\mathbf{k}}+i \delta\right)} .
$$

Then the single-hole Green's function in Eq[16 can be written as

$$
G_{h, \sigma}=G_{h, 1 \sigma}+\frac{N-1}{N} G_{h, 2 \sigma} \approx G_{h, 1 \sigma}+G_{h, 2 \sigma} .
$$

The single-hole spectral function, $A_{h, \sigma}=-(1 / \pi) \operatorname{Im} G_{h, \sigma}$ is now given by

$$
A_{h, \sigma}(\mathbf{k}, \omega)=A_{h, 1 \sigma}(\mathbf{k}, \omega)+A_{h, 2 \sigma}(\mathbf{k}, \omega) .
$$

The first contribution from the condensed bosons is

$$
\begin{aligned}
& A_{h, 1 \uparrow}(\mathbf{k}, \omega)=-\frac{1}{\pi}|x|^{2} \operatorname{Im} G_{f}(\mathbf{k}+\mathbf{Q}, \omega+i \delta), \\
& A_{h, 1 \downarrow}(\mathbf{k}, \omega)=-\frac{1}{\pi}|x|^{2} \operatorname{Im} G_{f}(\mathbf{k}-\mathbf{Q}, \omega+i \delta) .
\end{aligned}
$$

The second term is due to the interaction between the fermion and the uncondensed bosons and is given by

$$
\begin{aligned}
& A_{h, 2 \sigma}(\mathbf{k}, \omega) \\
& =-\sum_{\mathbf{q}} \int_{0}^{\omega} d y A_{f}(\mathbf{q}, y) A_{b, 2 \sigma}(\mathbf{q}-\mathbf{k}, y-\omega) \quad(\omega>0) \\
& =\sum_{\mathbf{q}} \int_{\omega}^{0} d y A_{f}(\mathbf{q}, y) A_{b, 2 \sigma}(\mathbf{q}-\mathbf{k}, y-\omega) \quad(\omega<0),
\end{aligned}
$$

where $A_{f}=-(1 / \pi) \operatorname{Im} G_{f}$ and $A_{b, 2 \sigma}=-(1 / \pi) \operatorname{Im} G_{b, 2 \sigma}$.

On the other hand, in the spin-liquid state (or the SRO phase), the first term in Eq28 vanishes because there is 


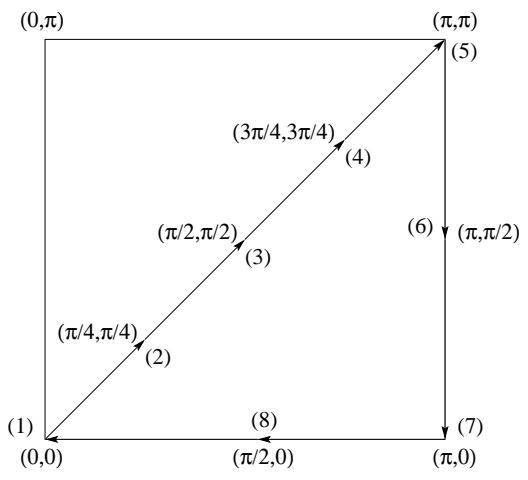

FIG. 5: Eight momenta in the principal Brillouin zone at which the spectral functions are evaluated.

no boson condensate and the spectral function is entirely given by the second term.

We will first numerically solve the Dyson equation for the fermion Green's function in both the LRO and the spin-liquid phases. Then the fermion spectral function can be convoluted with the boson spectral functions in the two phases to get the expression for the single-hole spectral function. The evolution of the resulting singlehole spectral function across the transition between the LRO and the spin-liquid phases is discussed in the next section.

\section{NUMERICAL RESULTS}

At first, the fermion Green's function is obtained by solving numerically the self-consistent Dyson equation on a discrete mesh of $\mathbf{k}$ and $\omega$ points. In order to do so, we have solved the saddle point equation of the large- $N$ mean-field theory for each set of $\kappa, J_{1}, J_{2}$, and use the mean field results as the input to the Dyson equation of the fermion Green's function. The full hole Green'sfunction is then computed by using Eqs 282930. The calculations are done with a finite lattice of size $8^{2}$. We have confirmed that the overall structure of the spectral function does not change when the size of the system is increased to $16^{2}$.

The evolution of the spectral function near the transition between the incommensurate LRO and the spinliquid phases, is examined at 8 different points in the phase diagram by following two different paths (see Fig 21. On both paths, the transition between two states is achieved by changing $\kappa$ for a given $J_{2} /\left(J_{1}+J_{2}\right)$. For each ground state, the spectral function is calculated at eight different momenta in the principal Brillouin zone. These eight momenta are shown in Fig [ When the spectral functions are plotted for these eight momenta, they will be arranged from bottom up in the order indicated by the bracketed numbers as shown in Fig [6 All other similar plots will follow the same convention.

As explained earlier, quantum fluctuations are stronger

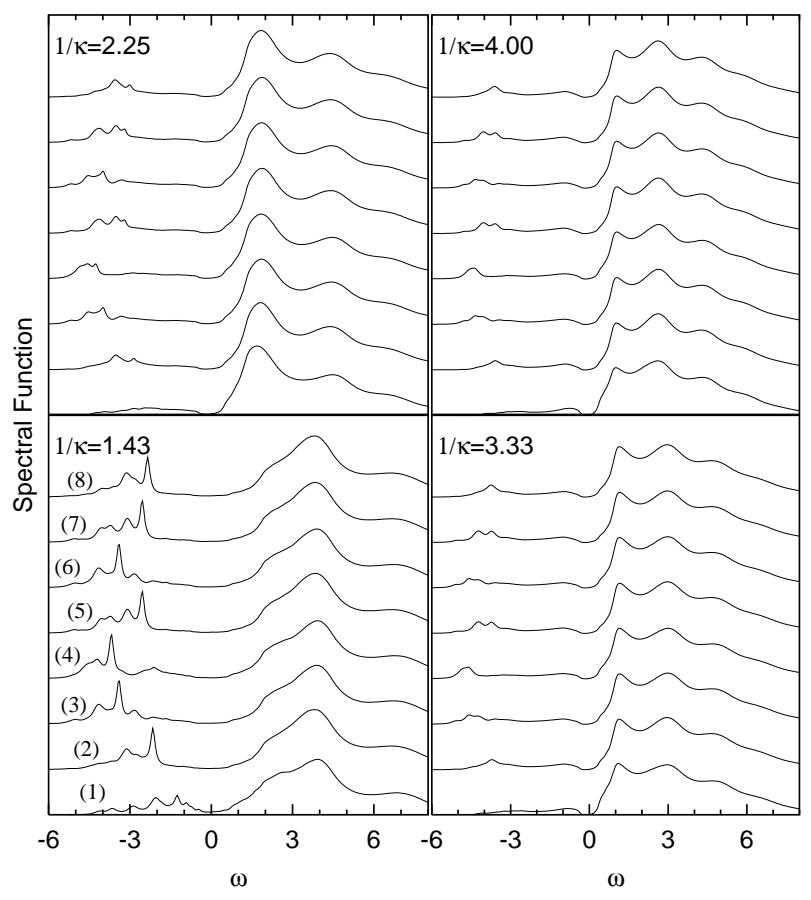

FIG. 6: Evolution of the spectral functions along path 1 in Fig. 2] $J_{1}=J_{2}=1.0$ and $t_{1} / J_{1}=t_{2} / J_{2}=2.5$.

for smaller values of $\kappa$. Thus, the transition from the incommensurate LRO phase to the spin-liquid state can be obtained by decreasing $\kappa$ for a given value of $J_{2} /\left(J_{1}+\right.$ $J_{2}$ ). We consider paths (1) and (2) corresponding to $J_{1} / J_{2}=1$ and $J_{1} / J_{2}=0.75$, respectively. The results for the four different values of $\kappa$ are shown in Fig 6 for path (1) and in Fig[7 for path (2).

The results of paths (1) and (2) are very similar. At large $\kappa$, there exists a sharp low-energy peak in the incommensurate LRO phase suggesting the existence of a coherent hole excitation or a quasiparticle. Notice that the incoherent continuum at higher energies are due to the existence of the 'unconfined' spinons. In the spinliquid phase, the quasiparticle peak completely disappears and only the incoherent broad continuum can be seen in the spectral function.

Notice that the spectral weight of the quasiparticle peak in the LRO phase decreases as the transition to the spin-liquid phase is approached and instead the spectral weight of the incoherent continuum grows. This is due to the fact that the spectral weight of the quasiparticle peak is proportional to the strength of the boson condensate or the magnetic order parameter that decreases as the transition point is approached. This can be observed in both Fig 6 and Fig [7 but is illustrated more clearly in the latter figure. In Fig 7 the strong quasiparticle peak seen when $\kappa=1$ diminishes as we approach the transition. The remnant of the peak is indicated by the arrows in the figure. Upon careful inspection, a slightly broad bump beside the peak consistently persists in all of the 


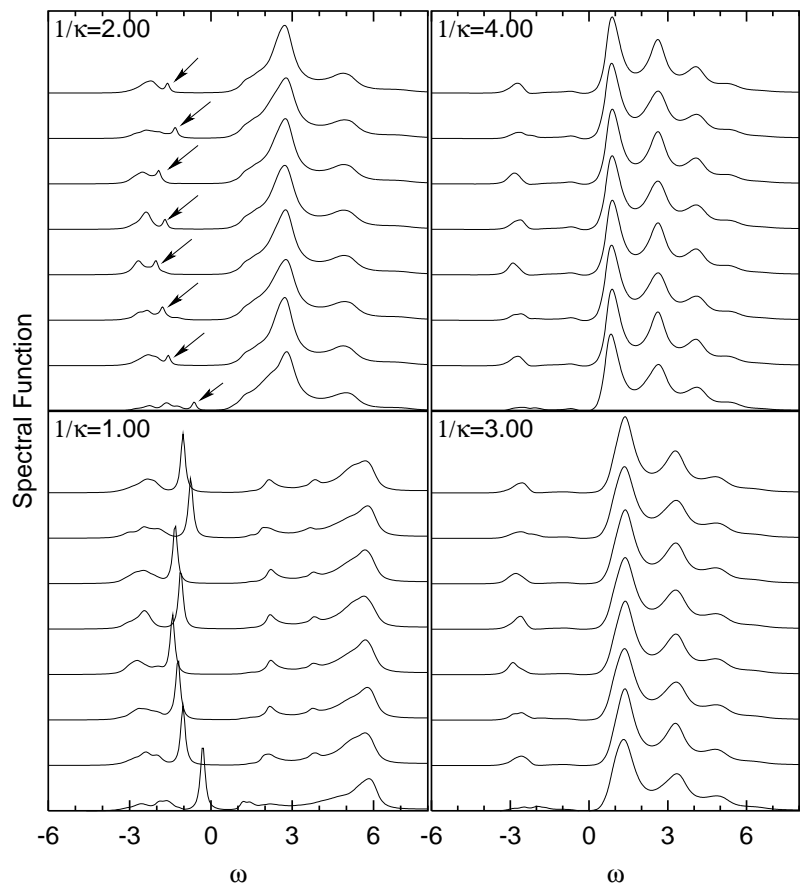

FIG. 7: Evolution of the spectral functions along path 2 in Fig. 2] $J_{1}=0.75, J_{2}=1.0$ and $t_{1} / J_{1}=t_{2} / J_{2}=2.5$.

plots indicating that it is a contribution from the unconfined spinons. One can observe in both figures that as the spectral weight of the quasiparticle peak shrinks, the remaining spectral weight gradually moves to the 'unconfined' spinons at higher energies.

The incoherent continuum in the SRO phase arises because the injected hole decays into its charge and spin degrees of freedom and they do not propagate together in a coherent fashion. The origin of the broad continuum in the LRO phase is basically the same; the 'unconfined' spinons make the motion of the hole incoherent. The reason why the low-energy quasiparticle peak survives in the LRO phase is that the density of states of the spinons becomes very small in the low energy limit so that the spin degree of freedom of the injected hole at low energy cannot efficiently decay into the spinon continuum.

We have also confirmed that varying the hopping amplitude, $t$, does not change the qualitative behaviour of the spectral function. The only change is that the bandwidth of the quasiparticle is increased as the hopping amplitude becomes bigger.

\section{SUMMARY AND CONCLUSION}

We investigated the single-hole spectral function in the anisotropic-triangular-lattice Heisenberg antiferromagnet. In particular, we studied the evolution of the single-hole spectral function across the transition between the incommensurate LRO phase and the spin- liquid state. This phase transition can be described by the large- $N \operatorname{Sp}(N)$ mean-field theory 14.15.16.17.18, where the $\operatorname{Sp}(N)$ analog of the spin magnitude, $\kappa$, can be adjusted to control the strength of quantum fluctuations. The motion of the single hole injected into the insulator is studied via the $t$ - $J$ type model in the slave fermion representation, where the electron is represented as the composite of a charge-carrying fermion and a spin-carrying boson. Taking into account the multiple interactions between the injected hole and the excitations of the underlying insulator in the non-crossing approximation, we evaluated the single-hole spectral function in both of the LRO and the spin-liquid phases.

It is found that, in the LRO phase, the quasiparticle peak exists at low energy and the broad incoherent background also arises at intermediate to high energies. This incoherent continuum arises due to the existence of 'unconfined' spinons in the large- $N$ mean-field theory. In principle, these spinons should be confined below a 'confinement' energy scale due to a fluctuation effect beyond the mean-field theory ${ }^{\underline{4}}$. Near the transition, however, the confinement energy scale becomes very small (in fact it is zero at the transition) so that the spinons at intermediate to high energies are 'almost' unconfined. In this respect, our calculation is particularly suited to describe the spectral function near the transition. As the transition to the spin-liquid state is approached, the spectral weight of the quasiparticle peak goes to zero and the incoherent continuum grows. Finally, in the spin-liquid phase, only the incoherent continuum survives in the spectral function. This is due to the fact that the injected hole can decay into the charge-carrying fermionic excitation and the spin-carrying spinon and the motions of these excitations are not coherent together.

In our studies, we neglected various vertex corrections, which may have lead to an underestimation of the coherence, especially in the spin-liquid phase. However, the recent exact diagonalization study of the spin-liquid state on the Kagomé lattice showed spectral functions that were indeed completely incoherent ${ }^{29}$, leading to the conclusion that the vertex correction would not change the qualitative behaviours obtained in our study.

Our work is relevant to possible ARPES experiments on Mott insulators on the anisotropic triangular lattice since the ARPES on the insulator can measure the singlehole spectral function ${ }^{24}$. In particular, notice that the neutron scattering experiment on $\mathrm{Cs}_{2} \mathrm{CuCl}_{4}$ reveals the existence of the spinon continuum at intermediate to high energies in the LRO phase near the transition as well as in the spin-liquid phase $\frac{12,13}{}$. The parameter $1 / \kappa$ in the large- $N$ theory plays the same role as the in-plane magnetic field in the experiment on $\mathrm{Cs}_{2} \mathrm{CuCl}_{4}$, where the transition between the two phases is achieved by changing the in-plane field or temperature. Thus we expect that, in light of our work, the ARPES experiments on such systems will provide independent confirmation of the spinon continuum discovered in the neutron scattering experiments on $\mathrm{Cs}_{2} \mathrm{CuCl}_{4}$ or in related systems. 
Acknowledgment: This work was supported by the NSERC of Canada, Canadian Institute for Advanced Re- search, and Canada Research Chair Program.

16 N. Read and S. Sachdev, Phys. Rev. Lett. 66, 1773 (1991).

17 S. Sachdev and N. Read, Int. J. of Mod. Phys. B 5, 219 (1991).

18 S. Sachdev, Phys. Rev. B 45, 12377 (1992).

19 C.-H. Chung, J.B. Marston, and S. Sachdev, Phys. Rev. B 64, 134407 (2001).

20 R. Moessner, O. Tchernyshyov, and S. L. Sondhi, cond-mat/0106286

21 J.-S. Bernier, C.-H. Chung, Y.B. Kim, and S. Sachdev, cond-mat/0310504

22 An earlier discussion of the incommensurate order on the anisotropic triangular lattice can be found in T. Jolicoeur, E. Dagotto, E. Gagliano and S. Bacci, Phys. Rev. B 42, 4800 (1990).

23 D. S. Rokhsar and S. A. Kivelson, Phys. Rev. Lett. 61, 2376 (1988); S. A. Kivelson, Phys. Rev. B 39, 259 (1989); N. Read and B. Chakraborty, Phys. Rev. B 40, 7133 (1989); R. A. Jalabert and S. Sachdev, Phys. Rev. B 44, 686 (1991); X.-G. Wen, Phys. Rev. B 44, 2664 (1991); T. Senthil and M. P. A. Fisher, Phys. Rev. B 63, 134521 (2001); C. Nayak and K. Shtengel, Phys. Rev. B 64, 064422 (2001); R. Moessner, S. L. Sondhi, and E. Fradkin, Phys. Rev. B 65, 024504 (2002); L. Balents, M. P. A. Fisher, and S. M. Girvin, Phys. Rev. B 65, 224412 (2002); E. Demler, C. Nayak, H.-Y. Kee, Y. B. Kim, T. Senthil, Phys. Rev. B 65, 155103 (2002).

24 B.O. Wells, Z.-X. Shen, A. Matsuura, D.M. King, M.A. Kastner, M.Greven, and R.J. Birgeneau, Phys. Rev. Lett. 74, 964 (1995).

25 M. Vojta, Phys Rev. B 59, 6027 (1999).

26 M. Azzouz and T. Dombre, Phys. Rev. B 53, 402 (1996).

27 C.L. Kane, P.A. Lee, and N. Read, Phys. Rev. B 39, 6880 (1989).

28 F. Marsiglio, A.E. Ruckenstein, S. Schmitt-Rink, and C.M. Varma, Phys. Rev. B 43, 10882 (1991).

29 A. Läuchli and D. Poilblanc, cond-mat/0310597
14 N. Read and S. Sachdev, Phys. Rev. B 42, 4568 (1990).

15 S. Sachdev, Phys. Rev. Lett. 66, 1773 (1991). 